\title{
The many facets of polyelectrolytes and oppositely charged macroions complex formation
}

\author{
Serge Ulrich, Marianne Seijo, Serge Stoll * \\ CABE (Analytical and Biophysical Environmental Chemistry), Department of Inorganic, Analytical and Applied Chemistry, \\ University of Geneva, Sciences II, 30 quai E. Ansermet, CH-1211 Geneva 4, Switzerland
}

Available online 2 October 2006

\begin{abstract}
Various models of the complex formation between polyelectrolyte chains and oppositely charged macroions are reviewed. In recent years, a great deal of knowledge of the multitude of possible polyelectrolyte conformations at the macroion surface has been accumulated, which consequently has led to increasing interest in using such complexes in the design of nanomaterials. This review focuses on key studies relating to the effects of various physico-chemical parameters on complex formation and areas for future research are identified.
\end{abstract}

(C) 2006 Elsevier Ltd. All rights reserved.

Keywords: Polyelectrolytes; Macroions; Complex formation; Overcharging; Weak and strong polyelectrolytes

\section{Introduction}

Bulk interactions between large polyelectrolyte chains (PEs) and oppositely charged macroions such as inorganic particles, micelles, globular proteins, macroions, are of great importance for many industrial processes such as water treatment as flocculant/water insoluble mixtures, powder processing as dispersion agents, or food technology as rheology modifiers. Many biomacromolecules, such as DNA, are also polyelectrolytes and the formation of complexes with proteins or membranes, for example, are expected to play critical roles in biological regulation processes with applications in therapeutic delivery systems. In addition the formation of complexes between PEs and macroions to the formation of building blocks is expected to find important applications in nanoscience. Owing to the practical and economical aspects for the rational use of $\mathrm{PE} /$ macroion mixtures, as well as the theoretical interest of such systems in soft condensed matter science, nanochemistry, biology and medicine, a better understanding of the key factors controlling the complexation between macroions and oppositely charged macromolecules is therefore important.

It should be noted here that for PE complexation we have to make distinction between strong and weak polyelectrolytes since adsorption of weak polyelectrolytes on surfaces is a more com-

\footnotetext{
* Corresponding author.

E-mail address: serge.stoll@cabe.unige.ch (S. Stoll).
}

plicated process owing to the fact that the linear surface charge density of the PE is strongly influenced by the charge connectivity along the chain, the $\mathrm{pH}$ of the solution, the solution ionic concentration and presence of an oppositely charged surface which is expected to influence the PE degree of dissociation.

This review focuses on research relating to the formation and structure of PEs and oppositely charged macroions complexes, based on the principle of electrostatic complexation, at the experimental, theoretical and computational level. In particular we focus on computer simulations since they allow treatments of polyelectrolyte adsorption on flat or curved macroion surfaces over a large range of parameters and in some situations fill the gap between existing theories and experiments.

\section{Experimental investigations}

A series of studies from Dubin illustrated the transition-like behaviour of the binding of polyelectrolytes to oppositely charged spherical macroions such as micelles, dendrimers and globular proteins [1-5]. The binding affinity of PEs was found to be strongly influenced by chain flexibility, charge mobility, and charge density on the macroion [6]. In particular it was shown that the critical surface charge density on the macroion required to bind the PE becomes greater at higher salt concentrations. Similarly, stiffer polymer chains were found harder to bind than the more flexible ones. More subtle effects seen for protein binding [7] were 
attributed to charge heterogeneity, which introduces repulsive forces between polyanions and protein negative domains and also alters the nature of the polyanion-binding site. Complexes involving polyelectrolyte-neutral block copolymers and oppositely charged micelles were recently investigated by Berret [8-12]. The structure of such complexes was elucidated by small-angle neutron scattering and it was found that these complexes exhibit a core-shell microstructure, where the core is a dense coacervate microphase of micelles surrounded by neutral blocks. With these results, they showed that the formation of the colloidal complex was in agreement with overcharging predictions i.e. the amount of PE needed to build the core-shell colloids always exceed the number that would be necessary to compensate the charge of the micelles. Controlled clustering of superparamagnetic macroions using such block copolymers was successfully explored for the design of new contrast agent for magnetic resonance imaging. Ilekti and coworkers [13] presented experimental results on the effects of PEs on surfactant aggregates by means of small-angle X-ray scattering. The authors considered a mixture of sodium polyacrylate and dilute micellar solutions of cetyltrimethylammonium bromide. When PEs were added to the dilute micellar solution, the micelles were stick together by polyion bridges in a concentrated phase.

\section{Theoretical modeling}

Boroudjerdi and Netz [14] reviewed the problem of complex formation by explicitly presenting the adsorption behavior of polyelectrolytes on macroions of different geometries. The relevance of salt, which modulates the electrostatic interactions between PEs and macroions was discussed by considering parameters appropriate for DNA-histone complexes which are the basic building blocks of the nucleosomal genetic structure. Both experiments and analytical theory demonstrated that the complex was only stable for intermediate salt concentration. For low salt concentration, difficulties to charge the polyelectrolyte due to strong charged monomers repulsions were found, that is detrimental to the full polyelectrolyte complexation at the surface of the macroion. For high salt screening, despite the fact that chain ionization is usually promoted, attraction forces between polyelectrolyte and macroion are strongly reduced and thus, is now detrimental to the formation of compact complexes. Analytical models also demonstrate that a multitude of structures ranging from tight complexes with the chain more or less regularly wrapped around the macroion, to open multileafed rosette like complexes can be achieved. Netz and Joanny [15] provided a full complexation phase diagram for a stiff PE in presence of an oppositely charged sphere, whereas Schiessel [16] provided scaling theories for the charged rosettes formation for the limits of high and low ionic concentrations, presented a full phase diagram as a function of the PE contour length and investigated the influence of the PE persistence length. Rosette structures were found quite robust against changes in the ionic strength.

Hence, an important feature in complex formation is the chain stiffness of PE which includes both chain stiffening due to electrostatic monomer/monomer repulsions and flexibility of the underlying chain backbone which is controlled by the local PE chemical structure. Manning [17] (as well as Dubin from an experimental point of view) clearly demonstrated that stiffer PEs are harder to bind than more flexible ones. For a given PE, the curvature of the macroion imposes stability limits on the bound state since it is harder to bind to a smaller macroion than to a larger one. An interesting situation dealing with the complexation of a long flexible PE was investigated by Nguyen and Shklovskii [18]. They demonstrated that PE binds the small macroions winding around them, while they repel each other and form almost periodic necklace. The effect of charge inversion on the complex structure was investigated in details as well as the influence of screening by monovalent salt. It was found that near the isoelectric point where total charges of macroions and PE are equal, necklaces condense into macroscopic bundles.

If electrostatic repulsions between charges on neighbouring monomers tend to decrease the effective charge of the PE, the situation is more complicated close to an interface with a subtract of low and high dielectric constant, since here the charge on each monomer is interacting with its neighbours but also with its own image charges and the image charges of all neighbours. Netz [19] investigated this phenomenon and found that at low-dielectricconstant substrates, one find a further charge decrease and repulsion from the interface, while at high-dielectric-constant substrates, the effective charge increases and the PE is attracted to the interface. He also investigated the interaction between two complexes, which gives rise to configurational changes of two complexes as they approach each other [20]. Recently, analytical solutions for quantities such as the critical adsorption radius and critical surface charge density were provided by Winkler and Cherstvy [21] in good agreement with computer simulations.

\section{Simulations}

There is nowadays a considerable amount of available contributions investigating the $\mathrm{PE} /$ macroion complexation by

Table 1

Equilibrated conformations of fully ionized $\mathrm{PE} /$ macroion complexes at ionic concentration $C_{i}=0.001 \mathrm{M}$

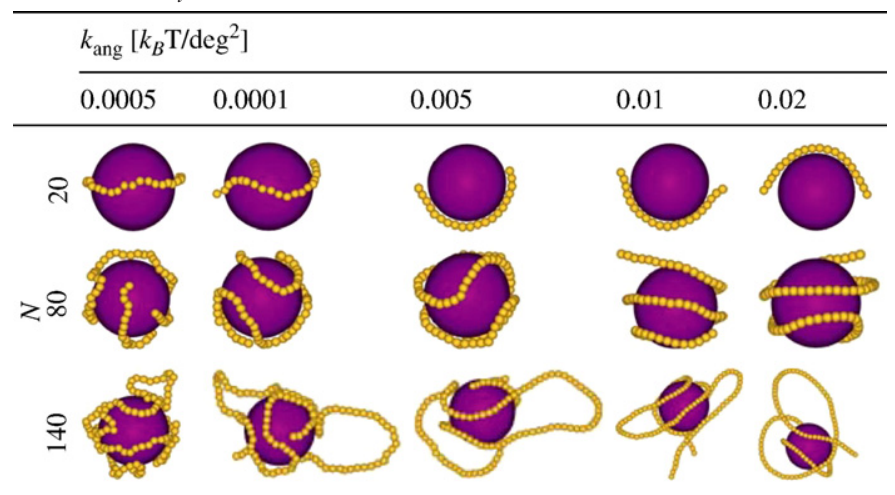

Macroion is characterized by a surface charge density equal to $+100 \mathrm{mC} / \mathrm{m}^{2}$ and a radius of $35.7 \AA$. Various polymer lengths $N$ and chain stiffness $k_{\text {ang }}$ (in $k_{\mathrm{B}} \mathrm{T}$ / $\mathrm{deg}^{2}$ units - the bending energy is given by $E_{\text {tor }}=\sum_{i=2}^{N} k_{\text {ang }}\left(\theta_{i}-\theta_{0}\right)^{2}$ where $\theta_{i}$ represents the angle formed by the vectors consecutive monomers $r_{i-1}-r_{i}$ and $r_{i+1}-r_{i}$ and $\left.\theta_{0}=180^{\circ}\right)$. Solenoid, tennis ball, tail and rosette conformations are achieved. Rosette conformations are characterized by extended loops whose size is decreasing with the increase of the PE flexibility. 
means of Monte Carlo [22-30•31-34•35-39], or Molecular Dynamic $[40,41]$ simulations. Considering one PE and one or several macroions, the effects on complexation of parameters such as the chain stiffness, chain length, ionic concentration, particle size and surface charge density just to quote a few were systematically investigated by Linse's group [27-32] and Stoll's group [24-26,33-36]. The effect of chain stiffness of a strong PE were first studied by Wallin and Linse [22] and then further investigated by Stoll and Chodanowski [26] and Akinchina and Linse [27]. Wallin and Linse [22] showed that PE adsorption decreases with the stiffness of the chain. Stoll and Chodanowski [26] showed that by increasing the chain stiffness, solenoid conformations could be obtained. They also investigated the effect of added salt on the formation of complexes between a flexible, semiflexible, and rigid PE and an oppositely charged spherical macroion and the adsorption/desorption transition. According to these studies $[22,26,27,36]$, a range of macromolecular structures such as tennis ball, solenoid, and multiloop or rosette were obtained (see Table 1).

Using numerical simulations, Kunze and Netz [42,43] also investigated the case of complexation of a semi flexible PE and an oppositely charged sphere. To answer the question of the agreement between simulations and experimental predictions, their parameters were adjusted to mimic the DNA/histone complex, and their results were in line with the expected trends. The effects of chain length were systematically investigated by Wallin and Linse [23]. For a high ratio of chain length upon macroion diameter, Stoll and coworkers $[24,25,34,36]$ found that only a few number of monomers is adsorbed to the macroion, forming an extended tails in solution (Fig. 1). Carlsson [44] and de

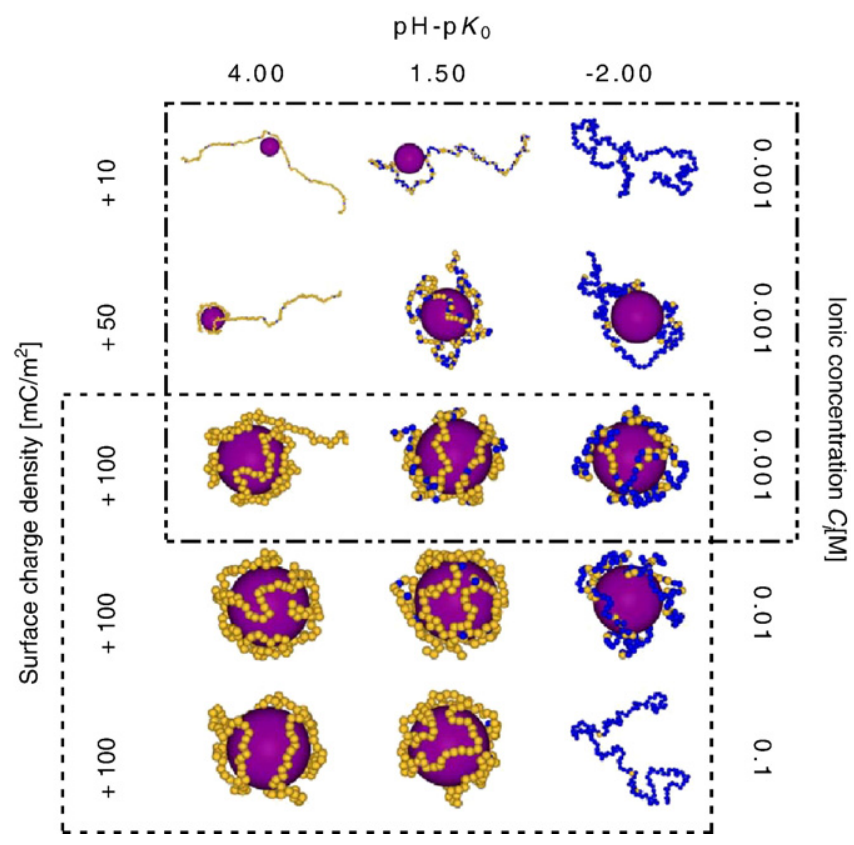

Fig. 1. Weak PE/macroion complex conformations considering a weak anionic

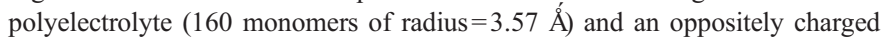
nanoparticle (radius $=35.7 \AA$ ). All pairs of charged monomers and nanoparticle interact with each other via a screened Debye-Hückel potential. The adsorption/ desorption limit, conformation and number of monomer in trains depend on ionic concentration, macroion surface charge density and $\mathrm{pH}$ of the solution.

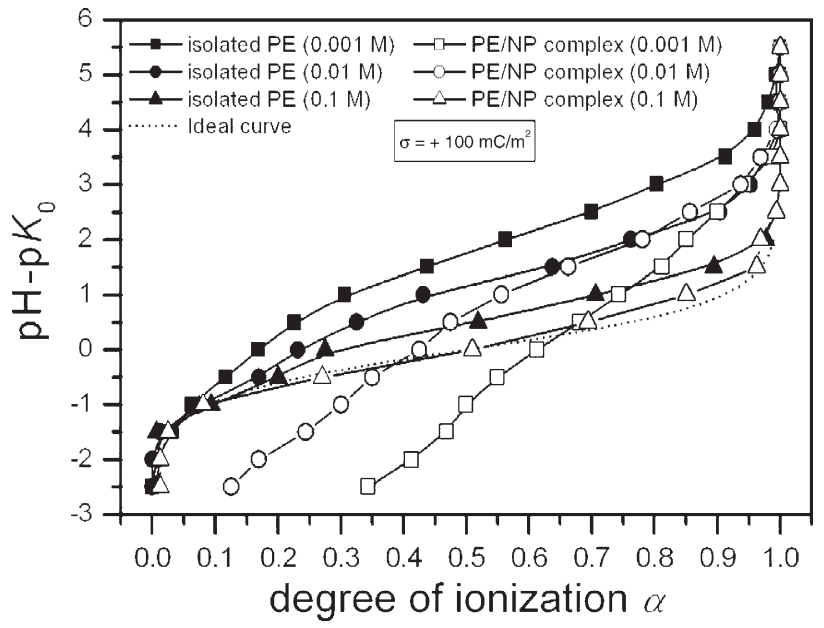

Fig. 2. Titration curves of a weak flexible isolated polymeric acid (160 monomers of radius=3.57 $\AA$ ) at various ionic concentrations ( $\mathbf{0 \Lambda}$ ). Differences between the acid/base properties of the chain and the corresponding ideal solution of monomer increases continuously with the PE degree of ionization. By adding an oppositely charged nanoparticle NP (radius=35.7 $\AA$ ), the resulting complex formation significantly changes the acid/base properties of PE chain $(\square \mathrm{O} \triangle$ ).

Vries [45] considered the effect of the discrete nature of the protein charge distribution on lysozyme/PE complexation. Simulations were performed with different protein charge distribution corresponding to solutions at different $\mathrm{pH}$. It was concluded that a protein model with a discrete charge distribution gave rise to more amount of adsorbed monomers because of the spatial charge-charge correlations.

It should be noted here that most of the computer simulations of PE complexation considered strong polyelectrolytes, although titrating or weak polyelectrolytes, which are an important class of PEs, were mainly studied by computer simulations for the isolated case [46-48]. The complex formation between macroions and a weak anionic PE was recently systematically investigated [3436] pointing out the importance of several competing effects on the PE-macroion complex structure and the influence of the macroion(s) on the PE titration curve as shown in Fig. 2. The adsorption/desorption limit and number of monomer in contact with the macroion surface was found to depend on ionic concentration, macroion surface charge density and solution $\mathrm{pH}$ as presented in Fig. 1. Then it was demonstrated that i) attractive interactions between the charged PE monomers and the macroion can profoundly affect the acid/base properties of a PE by promoting chain ionization (Fig. 2), ii) the ionic concentration decreases the attractive interaction between the $\mathrm{PE}$ and macroion but promotes $\mathrm{PE}$ ionization degree, and iii) chain stiffness promotes PE expansion and ionization but penalizes PE adsorption at the macroion surface. Once again it was found that the best conditions for the formation of strong complexes were achieved in the intermediate ionic concentration regime. Simulations also demonstrated that due to charge mobility, charges accumulate at the macroion surface suggesting that annealed PEs are expected to bind more strongly than quenched PEs of equivalent charge density in good agreement with experimental findings [6]. The influence of image forces on PE adsorption onto an oppositely charged surface was investigated by Messina and it was 
demonstrated that a dielectric discontinuity reduces the degree of $\mathrm{PE}$ adsorption and reduces charge inversion of the substrate [39]. They also largely contributed to the study of PE multilayering by means of numerical simulations $[37,38]$ which constitutes an important area for the development of nanocapsule technology based on layer-by-layer self-assembly.

\section{Conclusions}

A vast amount of scientific information on the formation of PE/ macroion complexes has now become available during the last years. Most of the possible structures have been theoretically and numerically identified and characterized, and significant advances have being made in understanding the role of the electrostatic attractive and repulsive interactions between PEs and macroions. Probably a more refined description of the macroions is still needed to account for surface charge heterogeneities, charge regulation processes, and different macroion geometries. Experimental, theoretical and simulation studies should now move to the investigation of the aggregate formation between such complexes or their interactions with biological membranes. The formation of core-shell polymer capsules by alternate adsorption of oppositely charged polyelectrolyte layers is also expected to represent an area for future research. Since the main driving force for all these processes is of electrostatic origin more refine interaction potentials could be used so as to take into account hydrophobic interactions as well as the role of hydrogen bonding which is expected to play a key role if one is interested in vivo phenomena.

\section{Acknowledgments}

The authors are grateful to the Swiss National Science Foundation- (Project No. 200020-101974/1) for the financial support.

\section{References and recommended readings}

[1] Dubin PL, The SS, McQuigg DW, Chew CH, Gan LM. Binding of polyelectrolytes to oppositely charged ionic micelles at critical micelle surface charge densities. Langmuir 1989;5:89-98.

[2] Zhang HW, Ray J, Manning GS, Morrefield CN, Newkome GR, Dubin PL. Interaction of a polycation with small oppositely charged dendrimers. J Phys Chem, B 1999;103:2347-54.

[3] Miura N, Dubin PL, Moorefield CN, Newkome GR. Complex formation by electrostatic interaction between carboxyl-terminated dendrimers and oppositely charged polyelectrolytes. Langmuir 1999;15: 4245-50.

[4] Feng XH, Dubin PL, Zhang HW, Kirton GF, Bahadur P, Parotte J. Critical conditions for binding of dimethyldodecylamine oxide micelles to polyanions of variable charge density. Macromolecules 2001;34: 6373 .

[5] Kayitmazer AB, Seyrek E, Dubin PL, Staggemeier BA. Influence of chain stiffness on the interaction of polyelectrolytes with oppositely charged micelles and proteins. J Phys Chem, B 2003;107:8158-65.

[6•] Cooper CL, Goulding A, Kayitmazer AB, Ulrich S, Stoll S, Turksen S, et al. Effects of polyelectrolyte chain stiffness, charge mobility, and charge sequences on binding to proteins and micelles. Biomacromolecules 2006;7:1025-35.The binding affinity of anionic polyelectrolytes for bovine serum albumin or for an oppositely charged micelle

\footnotetext{
- Of special interest.

$\bullet$ Of outstanding interest.
}

were clearly found to be strongly influenced by three polyelectrolyte structural parameters: chain flexibility, charge mobility, and charge density which were systematically investigated.

[7••] Cooper CL, Dubin PL, Kayitmazer AB, Turksen S. Polyelectrolyte-protein complexes. Curr Opin Colloid Interface Sci 2005;10:52-78. A very comprehensive review in the field of polyelectrolye-protein complexes.

[8] Berret JF, Cristobal G, Herve P, Oberdisse J, Grillo I. Structure of colloidal complexes obtained from neutral/poly-electrolyte copolymers and oppositely charged surfactants. Eur Phys J, E Soft matter 2002;9: 301-11.

[9] Berret J-F, Herve P, Aguerre-Chariol O, Oberdisse J. Colloidal complexes obtained from charged block copolymers and surfactants: a comparison between small-angle neutron scattering, cryo-TEM, and simulations. J Phys Chem, B 2003;107:8111-8.

[10] Berret J-F, Oberdisse J. Electrostatic self-assembly in polyelectrolyteneutral block copolymers and oppositely charged surfactant solutions. Physica B 2004;350:204-6.

[11] Berret J-F. Evidence of overcharging in the complexation between oppositely charged polymers and surfactants. J Chem Phys 2005;123: 164703-1-164709-10.

[12•] Berret J-F, Schonbeck N, Gazeau F, El Kharrat D, Sandre O, Vacher A, et al. Controlled clustering of superparamagnetic nanoparticles using block copolymers: design of new contrast agents for magnetic resonance imaging. J Am Chem Soc 2006;128:1755-61. The formation of new hybrid aggregates comprising charged block copolymers and nanoparticles with contrast properties in magnetic resonance.

[13] Ilekti P, Martin T, Cabane B, Piculell L. Effects of polyelectrolytes on the structures and interactions of surfactant aggregates. J Phys Chem, B 1999;103:9831-40.

[14] Boroudjerdi H, Kim YW, Naji A, Netz RR, Schlagberger X, Serr A. Statics and dynamics of strongly charged soft matter. Phys Rep 2005;416: 129-99.

[15] Netz RR, Joanny J-F. Complexation between a semiflexible polyelectrolyte and an oppositely charged sphere. Macromolecules 1999;32: 9026-40.

[16••] Schiessel H. The physics of chromatin. J Phys, Condens Matter 2003;15:R699-774. A simple system with a spherical particle and a semi-flexible polymer chain was adopted to capture the essential features of nucleosomes. Full phase diagram were presented.

[17] Manning GS. Simple Model for the Binding of a Polyelectrolyte to an Oppositely Charged Curved Surface. J Phys Chem B 2003;107: 11485-90.

[18•] Nguyen TT, Shklovskii BI. Complexation of a polyelectrolyte with oppositely charged spherical macroions: giant inversion of charge. J Chem Phys 2001;114:5905-16. The importance of the charge inversion on the complex structure is illustrated here.

[19] Netz RR. Charge regulation of weak polyelectrolytes at low-and highdielectric-constant substrates. J Phys, Condens Matter 2003;15: S239-44.

[20] Boroudjerdi H, Netz RR. Strongly coupled polyelectrolyte-macroion complexes. J Phys, Condens Matter 2005;17:S1137-51. The authors presented the corresponding phase diagram as a function of the separation distance of two complexes and the inverse Debye screening length $\kappa$ : They distinguished a phase where both complexes present the same structure (symmetric phase) from an other phase (asymmetric phase) where the complexes exhibit different structures because mutual interactions. The asymmetric phase had several conformations and as a result, a third phase was also put in evidence where one of the two chains was simultaneously adsorbed on both spheres (bridging phase).

[21] Winkler RG, Cherstvy AG. Phys Rev Lett 2006;96:066103-1-066103-4.

[22] Wallin T, Linse P. Monte Carlo simulations of polyelectrolytes at charged micelles. 1. Effects of chain flexibility. Langmuir 1996;12: 305-14.

[23] Wallin T, Linse P. Monte Carlo simulations of polyelectrolytes at charged micelles. 3. Effects of surfactant tail length. J Phys Chem, B 1997;101:5506-13. 
[24] Chodanowski P, Stoll S. Polyelectrolyte adsorption on charged particles: ionic concentration and particle size effects-a Monte Carlo approach. J Chem Phys 2001;115:4951-60.

[25] Chodanowski P, Stoll S. Polyelectrolyte adsorption on charged particles in the Debye-Hueckel approximation. A Monte Carlo approach. Macromolecules 2001;34:2320-8.

[26] Stoll S, Chodanowski P. Polyelectrolyte adsorption on an oppositely charged spherical particle. Chain rigidity effects. Macromolecules 2002;35:9556-62.

[27] Akinchina A, Linse P. Monte Carlo simulations of polyion-macroion complexes. 1. Equal absolute polyion and macroion charges. Macromolecules 2002;35:5183-93.

[28] Akinchina A, Linse P. Monte Carlo simulations of polyion-macroion complexes. 2. Polyion length and charge density dependence. J Phys Chem, B 2003;107:8011-21.

[29] Jonsson M, Linse P. Polyelectrolyte-macroion complexation. II. Effect of chain flexibility. J Chem Phys 2001;115:10975-85.

[30•] Jonsson M, Linse P. Polyelectrolyte-macroion complexation. I. Effect of linear charge density, chain length, and macroion charge. J Chem Phys 2001;115:3406-18. The complexation between a linear flexible polyelectrolyte and several oppositely charged macroions was examined by employing a simple model system with focus on the electrostatic interactions.

[31] Skepoe M, Linse P. Complexation, phase separation, and redissolution in polyelectrolyte-macroion solutions. Macromolecules 2003;36: 508-19.

[32] Skepoe M. Competition between a macroion and a polyelectrolyte in complexation with an oppositely charged polyelectrolyte. J Phys Chem, B 2004;108:5431-7.

[33] Laguecir A, Stoll S, Kirton G, Dubin PL. Interactions of a polyanion with a cationic micelle: comparison of Monte Carlo simulations with experiment. J Phys Chem B 2003;107:8056.

[34•] Ulrich S, Laguecir A, Stoll S. Complex formation between a nanoparticle and a weak polyelectrolyte chain: Monte Carlo simulations. J Nanopart Res 2004;6:595-603. The complex formation between an isolated weak polyelectrolyte and an oppositely charged nanoparticle were investigated using Monte Carlo simulations with screened Coulomb potentials in the grand canonical ensemble. The phase diagrams of complex conformations were also presented. It was shown that the polyelectrolyte conformation at the surface of the nanoparticle is controlled by the attractive interactions with the nanoparticle but also by the repulsive interaction between the monomers.

[35] Laguecir A, Stoll S. Adsorption of a weakly charged polymer on an oppositely charged colloidal particle: Monte Carlo simulations investigation. Polymer 2005;46:1359-72.
[36] Ulrich S, Laguecir A, Stoll S. Complexation of a weak polyelectrolyte with a charged nanoparticle. Solution properties and polyelectrolyte stiffness influences. Macromolecules 2005;38:8939-49.

[37] Messina R, Holm C, Kremer K. Polyelectrolyte multilayering on a charged sphere. Langmuir 2003;19:4473-82.

[38] Messina R. Polyelectrolyte multilayering on a charged planar surface. Macromolecules 2004;37:621-9.

[39] Messina R. Effect of image forces on polyelectrolyte adsorption at a charged surface. Phys Rev, E 2004;70: 051802-1-051802-9.

[40] Messina R, Holm C, Kremer K. Conformation of a polyelectrolyte complexed to a like-charged colloid. Phys Rev, E 2002;65: 041805-1-041805-4.

[41] Messina R, Holm C, Kremer K. Like-charge colloid-polyelectrolyte complexation. J Chem Phys 2002;117:2947-60.

[42] Kunze K-K, Netz RR. Salt-induced DNA-histone complexation. Phys Rev Lett 2000;85:4389-92.

[43] Kunze K-K, Netz RR. Complexes of semiflexible polyelectrolytes and charged spheres as models for salt-modulated nucleosomal structures. Phys Rev, E 2002;66: 011918/011911-010119/011928.

[44•] Carlsson F, Linse P, Malmsten M. Monte Carlo simulations of polyelectrolyte-protein complexation. J Phys Chem, B 2001;105: 9040-9. Complexation between a polyanion and a lysozyme were investigated by means of Monte Carlo simulations. The authors concluded the polyelectrolyte can bind to protein of the same charge sign if short-range interactions are included. When only electrostatic interactions are considered, a maximum in the number of monomers reaches a maximum value.

[45] de Vries R. Monte Carlo simulations of flexible polyanions complexing with whey proteins at their isoelectric point. J Chem Phys 2004;120: 3475-81.

[46] Ullner M, Jönsson B, Soederberg B, Peterson C. A Monte Carlo study of titrating polyelectrolyes. J Chem Phys 1996;104:3048-57.

[47] Ullner M, Jönsson B. A Monte Carlo study of titrating polyelectrolytes in the presence of salt. Macromolecules 1996;29:6645-55.

[48••] Ullner M, Woodward CE. Simulations of the titration of linear polyelectrolytes with explicit simple ions: comparisons with screened coulomb models and experiments. Macromolecules 2000;33:7144-56. Monte Carlo simulations have been extensively used by Ullner and coworkers to investigate different models for a linear, titrating polyelectrolyte in both salt-free environment [46] or in the presence of salt [47], and using explicit simple ions [48]. 